\title{
An Overview of Thermomechanically Heat-treated Nickel-Titanium Alloy Used in Endodontics
}

\author{
Loganathan Ashok ${ }^{1}$, Vineesh Krishnan ${ }^{2}$, Rajesh S Nair ${ }^{3}$, Mano C Angelo ${ }^{4}$
}

\begin{abstract}
The use of nickel-titanium (NiTi) alloys created a revolutionary impact in endodontic treatments. Shape memory and superelastic property of an $\mathrm{NiTi}$ alloy make the instrument more flexible, which improves the quality of the treatment by reducing the procedural errors. Even though these instruments show advanced metallurgical properties, it can lead to fracture due to fatigability. To improve the fracture resistance and enhance the clinical performance, various thermomechanical heat treatments had been introduced to these NiTi instruments. NiTi alloy undergoes phase transformation on heat treatment to upgrade the mechanical properties. This review article appraises the improved metallurgical properties of NiTi alloy by various thermomechanical heat treatment processes in addition with discussion of recently introduced thermomechanically altered NiTi endodontic instruments.
\end{abstract}

Keywords: Austenite, Cyclic fatigability, Edge endo files, Martensite, Nickel-titanium alloy, Phase transformation, R-phase.

Conservative Dentistry and Endodontic Journal (2019): 10.5005/jp-journals-10048-0047

\section{INTRODUCTION}

Nickel-titanium alloys ( $\mathrm{NiTi}$ ) have been popularly used in endodontic practice because of its more desirable and enhanced metallurgical properties over stainless steel instruments. Shape memory and superelastic property of $\mathrm{NiTi}$ alloy allow the material more flexible, which makes a significant improvement in working of rotary endodontic instruments. Usage of NiTi alloys in enginedriven endodontic instruments will reduce the procedural errors when compared to stainless steel instruments. In spite of advanced metallurgical properties, NiTi instruments still prone for fracture during root canal instrumentation. Two types of fracture can occur in NiTi instruments, flexural fracture and torsional fracture. ${ }^{1}$ During instrumentation in curved canals, NiTi instruments undergoes flexural fracture due to increased cyclic fatigability. Torsional fracture occurs when repeated loading and unloading of instrument leads to repetitive phase transformation within the material. Application of stress over the instrument will lead to microstructural changes that in turn cause phase transformation, which can be controlled by several thermomechanical treatments of the alloy. The recently introduced thermomechanically treated $\mathrm{NiTi}$ instruments show improved fracture resistance and it has created a revolutionary impact on the mechanical properties, which improves the clinical performance in rotary endodontics.

\section{Nickel-Titanium Alloys}

$\mathrm{NiTi}$ alloy composed of nickel (56\%) and titanium (44\%). NiTi alloy was named NITINOL after it was developed by the Naval Ordinance Laboratory. Alloy exists in two different phases depending on crystal structure.

- Austenitic phase which is a parent phase with cubic B2 crystal structure.

- Martensitic phase with monoclinic B19 crystal structure.

When stress is applied, it undergoes phase transformation from austenitic phase to martensitic phase, which exhibits shape memory and superelastic property that ascertain the uniqueness of the alloy. Shape memory effect is the ability of the deformed
${ }^{1}$ Department of Conservative Dentistry and Endodontics, Sree Mookambika Institute of Dental Sciences, Coimbatore, Tamil Nadu, India

${ }^{2-4}$ Department of Conservative Dentistry and Endodontics, Sree Mookambika Institute of Dental Sciences, Kanyakumari, Tamil Nadu, India

Corresponding Author: Loganathan Ashok, Department of Conservative Dentistry and Endodontics, Sree Mookambika Institute of Dental Sciences, Coimbatore, Tamil Nadu, India, Phone: +91 9003308879 , e-mail:drashok7993@gmail.com

How to cite this article: Ashok L, Krishnan V, Nair RS, et al. An Overview of Thermomechanically Heat-treated Nickel-Titanium Alloy Used in Endodontics. Cons Dent Endod J 2019;4(2):34-38.

Source of support: Nil

Conflict of interest: None

material to revert to its original shape on heating due to phase transformation of stable-deformed martensite to stable austenite phase. Superelastic effect is completely recoverable elastic state between stable austenite to stress-induced martensite phase due to phase transformation. ${ }^{2}$ This molecular transition allows the material to become flexible without undergoing permanent deformation.

Conventional NiTi wires transform from parent austenitic phase to distorted rhombohedral crystal structure on heating under certain conditions. This phase is called intermediate R-phase.

Austenitic alloy is hard when compared to martensitic alloy and shows superior superelastic property. Martensitic alloy is soft and ductile and shows shape memory effect, because of this property, it exhibits increased cyclic fatigue resistance than austenitic alloy (Fig. 1).

If the transformation temperature is above austenitic finish temperature $\left(A_{\mathrm{f}}\right)$, then the alloy is in austenitic state. On cooling, if the temperature goes below the martensitic finish temperature $\left(M_{\mathrm{f}}\right)$, then the alloy is in martensitic phase or daughter phase. ${ }^{2}$ This transformation is also called as military transformation. 


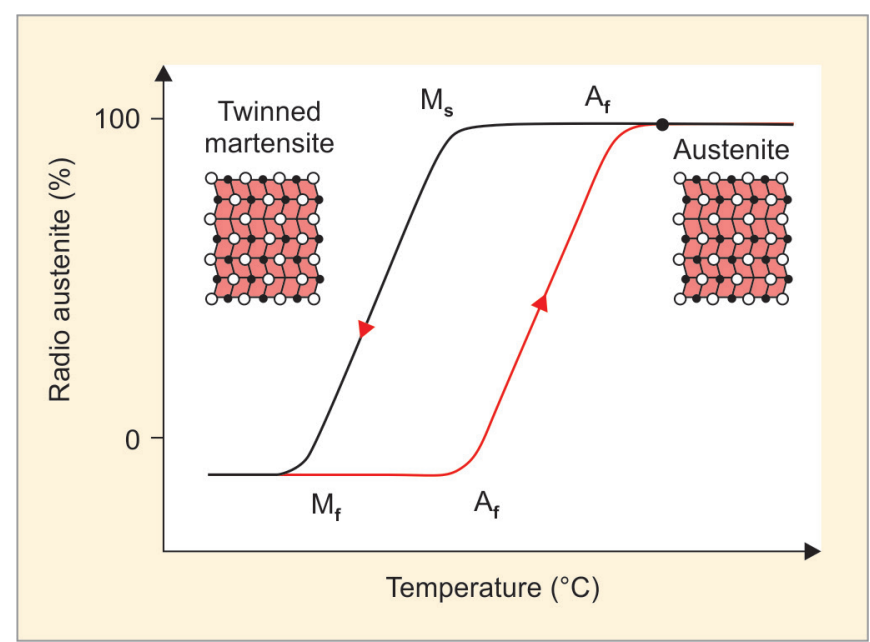

Fig. 1: Hysteresis of austenitic (parent) phase to martensitic (daughter) phase transformation (Courtesy: Zupanc J. Int Endod J 2018;51: 1088-1103)

Shear type process induced in the martensitic phase of an alloy, which gives rise to twinned martensite that forms the structure of a closely arranged hexagonal lattice. ${ }^{3}$ The original martensite shape can be deformed easily to a single orientation structure detwinned martensite by a process called detwinning. Martensitic phase possesses more ductile behavior than the austenite phase. The deformation can be reversed by heating the alloy above the temperature transformation range called reverse temperature transformation range (RTTR).

The thermoelastic behavior of a martensitic alloy can thrive either by a temperature variation called thermally induced martensite (TIM) or by the application of stresses which in turn result into strains is called strain-induced martensite (SIM) (Fig. 2).

Modulus of elasticity describes the stiffness of the material. The modulus of elasticity of martensitic alloy is 30-40 GPa which is lower than that of austenite, which shows the modulus of elasticity in the range of $80-90 \mathrm{GPa} .{ }^{2} \mathrm{R}$-phase shows modulus of elasticity lower than that of martensite.

\section{Fracture of Niti Alloys}

To minimize the iatrogenic procedural errors during root canal instrumentation, the use of stainless steel instruments had overcome by NiTi alloy instruments. Despite its flexibility, fracture of these instruments is one of the major problems in clinical practice. Reason for fracture is mainly due to incorrect or over usage of instruments. ${ }^{4}$ Two types of fracture can occur in NiTi instruments: (1) torsional fracture and (2) flexural fracture. Torsional fracture usually is the result of an intense tensional force that has been applied to the instrument. This means the continuous loading and unloading of instruments produces a high stress tension that may be superior to the $8 \%$ allowed by the alloy that causes unrecoverable plastic deformation and leads to fracture. ${ }^{1,5}$

Flexural fracture occurs due to cyclic fatigue of the instrument. When the instrument is subjected to repeated cycles of compression and tension, cyclic fatigue failure occurs. According to Martin and DiBernardo, after loading is applied to the instrument and analyzed using scanning electron microscope (SEM), the files begin to show irreversible microcrack formation on the alloy crystallographic structure. These microcracks lead to crack propagation process and contribute to failure. ${ }^{5}$

Phase transformation behavior plays an important role in mechanical properties of $\mathrm{Ni}-\mathrm{Ti}$ instruments and is easily influenced

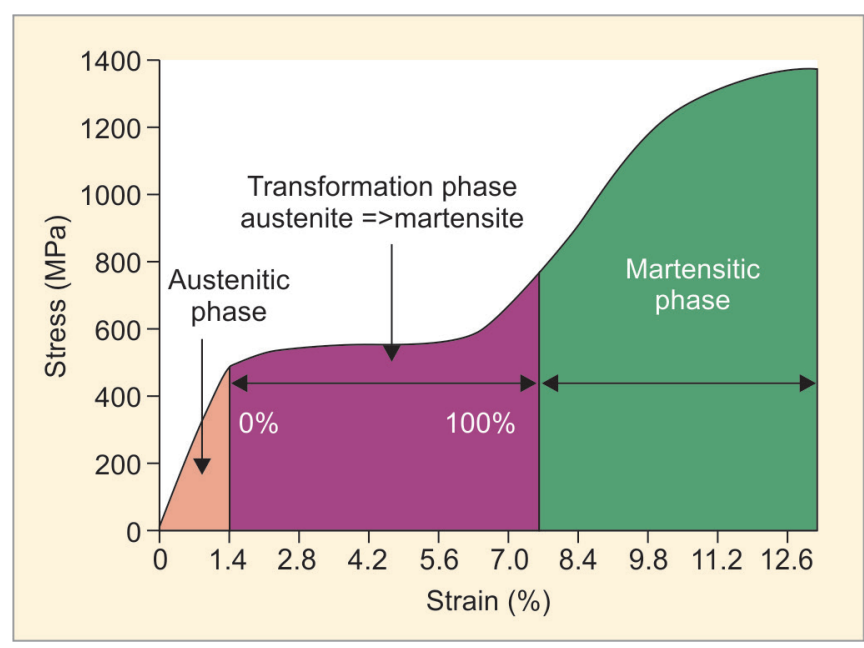

Fig. 2: NiTi phase transformation (Courtesy: Thompson SA. Int Endod J 2000;33:297-310)

by factors including chemical composition, heat treatment, and method of manufacturing. Flexibility of the instrument can be increased by phase transformation on heat treatment that can improve the metallurgical properties of NiTi files by increasing the cyclic fatigue resistance. ${ }^{6}$

Electropolishing is an electrochemical process of removing the surface irregularities and microcracks formed during grinding process. This will increase the fracture resistance and resistance to corrosion of the instrument. ${ }^{7}$

\section{Austenitic Alloy}

High flexibility of the instruments is one of the major advantages of $\mathrm{NiTi}$ alloys. When stress is applied to the instruments, it possesses superelastic property that can undergo reversible deformation of the instrument. To utilize superelastic property in NiTi endodontic instruments, the alloy should be in parent austenitic phase. ${ }^{8}$

\section{Conventional Niti Wires}

Conventional $\mathrm{NiTi}$ alloys are in the austenite phase at room temperatures. Activation of austenitic NiTi produces an elastic deformation that follows a linear stress/strain function. If stressinduced deformation increases, the superelastic deformation appears, whereas strain remains constant. This superelastic behavior is a direct consequence of the martensitic transformation, which occurs at the crystallographic level. Conventional NiTi instruments include Mtwo, OneShape, ProFile, and ProTaper Universal.

\section{M-WIRE}

A proprietary thermomechanical processing can be done to improve the cyclic fatigue resistance of the rotary $\mathrm{NiTi}$ instruments and to develop $M$-wire technology. Because of its unique nanocrystalline martensitic microstructure, $\mathrm{M}$-wire have higher strength and wear resistance than similar instruments made of conventional superelastic NiTi wires (Ye and Gao). ${ }^{9}$ According to Alapati et al., the austenite finish temperature of $\mathrm{M}$-wire was found to be around $43-50^{\circ} \mathrm{C}$ and this clearly shows it was well above austenitic finish temperature of conventional NiTi and body temperature, indicating that under clinical conditions, $\mathrm{M}$-wire is not completely composed of austenite. ${ }^{10} \mathrm{M}$-wire contains phases that are in both the deformed and microtwinned martensitic, R-phase, and are austenite whilst maintaining a pseudoelastic state. 
The examples of M-wire instruments include Dentsply's ProFile GT Series X, ProFile Vortex, ProTaper Next files, Path Files, WaveOne, and Reciproc (VDW, Munich, Germany). The R-phase and martensitic phase elastic modulus is lower than that of austenitic phase, and because of the presence of these phases in M-wire, it tends to be more flexible, thus shows improved cyclic fatigue resistance. The M-wire had physical and mechanical properties that can make the instruments more flexible and fatigue resistant than those made with conventionally processed NiTi wires. ${ }^{11}$

\section{R-PHASE}

The R-phase is an intermediate phase, a rhombohedral distortion of the cubic austenitic phase (Fig. 3), with one of the four directions of the cubic austenite stretched. ${ }^{12}$

The austenite to R-phase transformation is martensitic and is easily reversed by heating. Further, it introduces very small volume changes and can be self-accommodated by combining twin-related variants. This transformation is thermoelastic and has all the typical characteristics of a shape memory alloy, including shape memory and superelasticity (Fig. 3).

Leandro and his colleagues evaluated the mechanical property of R-phase using finite element analysis (FEA). They concluded that the R-phase instrument showed the highest flexibility when compared with other instruments. The R-phase demands a lower moment to be applied on an instrument during bending until $45^{\circ}$. The R-phase instrument shows the highest angular deflection in response to a torque of $3 \mathrm{~N} / \mathrm{mm}$ when torsional force is applied. This shows that the presence of R-phase in the alloy increases the flexibility but decreases the torsional stiffness of the endodontic instrument. The results from FEA study shows that the R-phase instrument presents the lowest stress values under bending, which is expected when a lower moment is required to bend an instrument. Thus, the same material presented stresses only on the surface during the torsional simulation. In spite of the larger deformation imposed on this instrument during torsion, its core is preserved from the damaging effects of this loading condition. ${ }^{13}$ R-phase instruments show similar cyclic fatigue resistance in comparison with those made of M-wire. ${ }^{14}$

Twisted file, twisted file adaptive, and K3XF (not twisted) are the examples of R-phase wires.

\section{Martensitic Alloy}

The unique characteristic of NiTi alloy that when it is cooled through a critical transformation temperature range (TTR), changes in modulus of elasticity, yield strength, and electric resistivity occur as a result of changes in electron bonding (Fig. 4).

By decreasing the temperature through this range, which leads to a change in the crystal structure is known as the martensitic
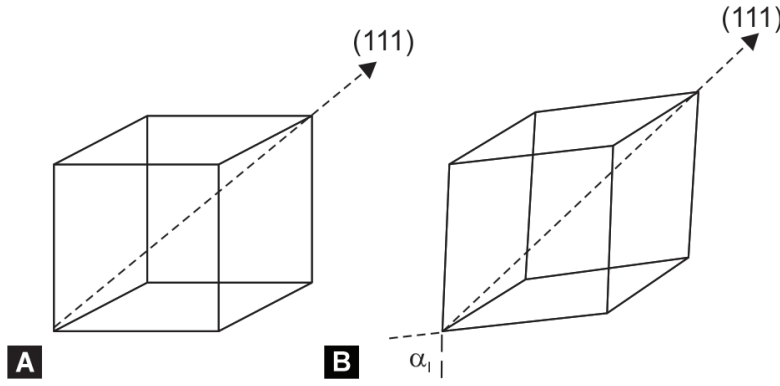

Figs 3A and B: (A) Cubic B2 austenitic phase; (B) Distorted rhombohedral R-phase (Courtesy: Duerig TW, Bhattacharya K. Shap Mem Superelasticity 2015;1:153-161) transformation. The amount of this transformation is a function of the start $\left(M_{\mathrm{s}}\right)$ and the finish $\left(M_{\mathrm{f}}\right)$ temperatures. This is the reason for the alloy possesses shape memory effect. Shear type of process induces the transformation that occurs in the alloy, which leads to change in its metallurgical properties, which forms a new phase called the martensitic or daughter phase which in turn gives rise to twinned martensite that forms the structure of a closely packed hexagonal lattice. This martensite shape can be deformed easily to a single orientation by a process known as detwinning to detwinned martensite when there is a "flipping over" type of shear. ${ }^{3}$ The additional property of the instrument in deformed martensitic phase is if the alloy is heated beyond the austenite finish temperature (e.g., autoclaving), it will return back to its original shape by returning to the primary austenitic state. Martensitic phase of NiTi alloy is more soft and ductile than the austenite phase. ${ }^{2}$

\section{WIRE}

CM wire was introduced by DS Dental in 2010. To increase the flexibility, reduce the shape memory, raise the transformation temperatures $\left(A_{\mathrm{f}}\right.$ to about $\left.50^{\circ} \mathrm{C}\right)$, and to obtain stable martensite at the body temperature of the NiTi alloy, a thermomechanical heat treatment is processed over the alloy. According to Testarelli et al., the nickel composition of CM wires has lower percentage in weight when compared to conventional NiTi alloy. CM wires exhibit martensitic phase with varying amounts of austenite and R-phase. The controlled memory effect helps the endodontic instrument to retain the shape of the canal even when it is taken out from the canal. This property helps avoid procedural errors such as ledge formation, transportation, and perforations. The extreme flexibility and less taper of these files show increased fatigue resistance in curved canal cases. ${ }^{15}$ Hyflex CM, Hyflex EDM, and Thypoon Infinite Flex NiTi files are the examples of CM wire.

\section{Hyflex CM}

The $A_{f}$ temperature of $C M$ wire (Hyflex $C M$ and Typhoon $C M$ ) is around $47-55^{\circ} \mathrm{C}$, which is above the intracanal temperature. ${ }^{16} \mathrm{Hyflex}$ $\mathrm{CM}$ wires show increased flexibility, high fatigue resistance, and improved cutting efficiency when compared to electropolished and conventional NiTi instruments. ${ }^{2,17}$ In the study of Shen et al., the higher $A_{f}$ temperature of $C M$ instruments was consistent with a mixture of austenite, $\mathrm{R}$ phase, and martensite structure. Hyflex

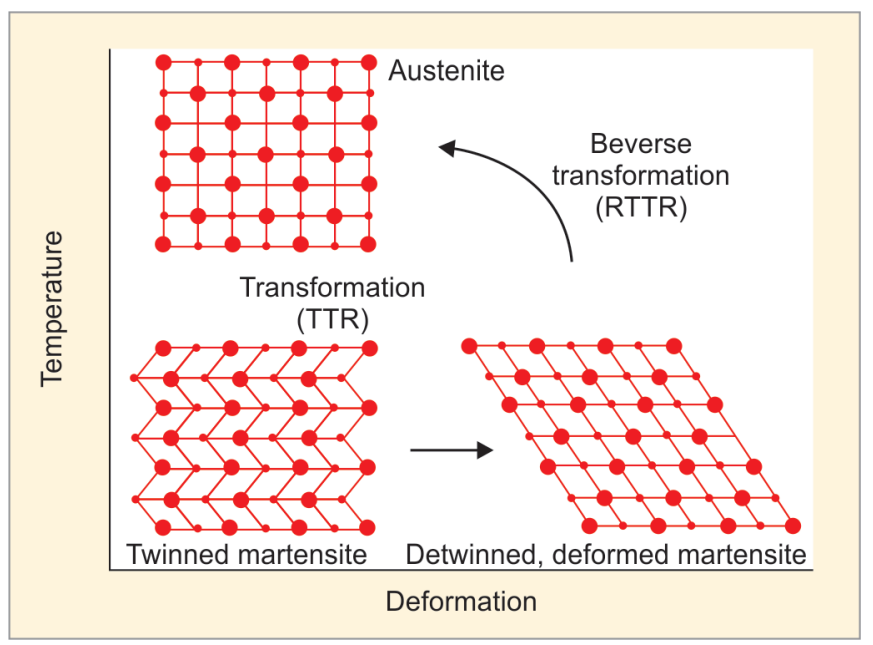

Fig. 4: Martensitic transformation on NiTi alloy (Courtesy:Thompson SA. Int Endod J 2000;33:297-310) 
CM files have unique characteristic of rewinding of the file after autoclaving, and if the file does not rewind, it indicates that it needs to be discarded. ${ }^{16,17}$

\section{Hyflex EDM}

Hyflex electrical discharge machining (EDM) is manufactured using the technique of EDM. Electrical discharge machining is also known as spark machining or spark eroding. It is a noncontact thermal erosion process used to machine electrically conductive materials using controlled electrical discharges to attain a desired shape. The electrical sparks used as a cutting tool cause a local melting and erode small portions of workpiece to produce crater-like surface finish. Both cutting tool and workpiece embedded in a dielectric liquid and voltage are applied. The metal removal is accomplished by pulsed electrical discharge. The ionized dielectric liquid removes the metal and produces nondirectional surface finish. This avoids the early instrument failure that results from conventional grinding techniques. After cutting and cleaning, the instrument placed in a ultrasonics acid bath and it is heat treated at temperature ranging between $300^{\circ} \mathrm{C}$ and $600^{\circ} \mathrm{C}$ for 10 minutes to 5 hours. The $A_{f}$ temperatures of EDM files is around $52^{\circ} \mathrm{C}^{1,2,15}$ According to Shen et al., the fatigue resistance of EDM files is higher than that of CM files. ${ }^{18}$ The metallographic analysis of new and used files shows a homogenous structure and it is mostly composed of lenticular martensite grains and some residual austenite (Pirani), and it shows $700 \%$ higher fatigue resistance than CM instruments. ${ }^{19}$

\section{Gold-treated and Blue-treated INSTRUMENTS}

To overcome the defects occurring during machining process and to modify the crystalline phase structure, new heat treatment method had been recently introduced called postmachining heat treatment. These instruments undergo proprietary thermomechanical heat treatment after grinding process. ${ }^{11}$ The first endodontic instrument possessing a distinctive blue color was ProFile Vortex Blue. Titanium oxide layer on the surface after machining process is responsible for distinctive blue color. Commercially, there are two gold-treated instruments, and two blue-treated instruments are available. In that, profile vortex blue and protaper gold are used in rotary. Reciproc blue and WaveOne gold are used in reciprocating motion. The $A_{\mathrm{f}}$ of blue treated instruments is around $38.5^{\circ} \mathrm{C}$ and $M_{\mathrm{s}}$ is around $31^{\circ} \mathrm{C} .{ }^{16}$ The $A_{f}$ of gold-treated instrument is around $50^{\circ} \mathrm{C}$, which indicates that these instruments mainly contain martensite or R-phase under clinical conditions. According Gao et al., all postmachining heat-treated instruments (gold and blue treated) shows enhanced flexibility and fatigue resistance. ${ }^{20}$ WaveOne gold undergoes special heat treatment before and after machining process. Superelastic NiTi alloy is subjected to heat treatment under constant strain in a temperature range of about $410-440^{\circ} \mathrm{C}$. After griding the working portion of the file, the finished instrument is heat treated a second time in a range of $120-260^{\circ} \mathrm{C}$. The $A_{\mathrm{f}}$ temperature of WaveOne gold is around $40-60^{\circ} \mathrm{C}$. It has the advantage that the gold technology increases the flexibility and strength of the instrument. Torsional resistance of WaveOne Gold is enhanced by the off-centered parallelogram-shaped cross-section design.

\section{Recently Introduced Thermomechanically Altered Niti files}

\section{Max Wire}

First endodontic nickel-titanium instrument that combines the property of superelasticity and shape memory effect. These instruments contain both martensitic $\left(20^{\circ} \mathrm{C}\right)$ and austenitic $\left(35^{\circ} \mathrm{C}\right)$ phases. Currently, two max wire (martensite-austenite-electropolish file X) instruments are available commercially XP-endo shaper and XP-endo finisher. These files are introduced by FKG Dentaire in 2016. Max wire instruments possess martensitic stable phase at room temperature. When it is placed inside the canal due to intracanal temperature changes, it transforms the phase into austenitic state. Thus, it exhibits both shape memory and superelastic property and changes its shape according to change in intracanal temperature. These instruments show improved torsional resistance during preparation in curved canal. On comparison with TRUShape instruments, XP-endo shaper instruments showed a higher cyclic fatigue resistance and angle of rotation to fracture but lower torque to failure. ${ }^{21}$ The $A_{f}$ temperature of XP-endo shaper files is around $35^{\circ} \mathrm{C}$. XP endo finisher is a new type anatomical finishing file and consists of a nontapered rotary file of size 25 . XP endo finisher $R$ is a variation of XP endo finisher file designed for retreatment cases. ${ }^{22}$

\section{K3 XF}

To increase the cyclic fatigue resistance, R-phase technology had been introduced in manufacturing of NiTi endodontic instruments. K3XF was developed by SybronEndo in 2011 which takes advantage of R-phase technology. It is fabricated similar to K3 files, but instead of twisting process, these files undergo grinding to attain a desired shape. K3XF files are subjected to a special heat treatment after the grinding process, which enhances the flexibility and strength and also modifies the crystalline structure of the alloy to accommodate some of the internal stress. K3XF instruments have an $A_{f}$ temperature below the body temperature $\left(37^{\circ} \mathrm{C}\right)$. Therefore, it has an austenite structure at body temperature and would exhibit a superelastic property during clinical application. The postmachining heat treatment processing used for K3XF modifies the transformation temperature by releasing crystal lattice defects and diminishing internal strain energy. ${ }^{1,23}$

\section{Edge Endo Files}

EdgeFile (EdgeEndo, Albuquerque, NM, USA) is made of an annealed heat-treated NiTi alloy brand named fire-wire. According to manufacturer, edge endo files show extreme durability, incredible flexibility, and strong enough to resist forces acting on torsion. Edge endo produces three different file systems $X 3, X 5$, and $X 7 .{ }^{24} X 3$ files are compatible with the settings of ProTaper and ProTaper Next (Dentsply Tulsa Specialties, Tulsa, OK, USA) rotary file systems. X5 files are compatible with GT and GT Series X (Dentsply) rotary file systems. X7 files are compatible with Vortex, ProFile (Dentsply), K3 (SybronEndo, Orange CA), EndoSequence (Brasseler USA, Savannah GA), TF (SybronEndo), and other similar 0.04/0.06 taper rotary file systems. According to Hansen, X7 files had shown extreme strength and flexibility on torsion and bending test. ${ }^{25}$

Examples: FlexiCON ${ }^{\mathrm{TM}}$, SmartTrack ${ }^{\mathrm{TM}}$, and Neoendo flex files.

\section{Conclusion}

Evident changes are documented in the metallurgical properties of $\mathrm{NiTi}$ alloys due to thermomechanical heat treatment. Recent advancements in the manufacturing process of NiTi alloys will enhance the quality of endodontic treatment by the development of newer endodontic instruments with superior mechanical properties. Postmachining thermomechanical heat treatment will increase durability of the instrument by increasing the flexural/ cyclic fatigue resistance. Superelasticity and shape memory effect of the NiTi instruments will result from phase transformation on 
heat treatment, which offered an promising result in enhancement of fatigue resistance during root canal preparation in complicated curved canals. In future, further research has to be needed for heat treatment of retreatment file systems and to improve the efficiency, safety, and quality of endodontic instruments.

\section{References}

1. Srivastava S, Alghadouni MA, Alotheem HS. Current strategies in metallurgical advances of rotary NiTi instruments: a review. J Dent Health Oral Disord Ther 2018;9(1):00333. DOI: 10.15406/ jdhodt.2018.09.00333.

2. Zupanc J, Vahdat-Pajouh N, Schäfer E. New thermomechanically treated NiTi alloys - a review. Int Endod J 2018;51(10):1088-1103. DOI: 10.1111/iej.12924.

3. Thompson SA. An overview of nickel-titanium alloys used in dentistry. Int Endod J 2000;33(4):297-310. DOI: 10.1046/j.13652591.2000.00339.x.

4. Gambarini G. Cyclic fatigue of ProFile rotary instruments after prolonged clinical use. Int Endod J 2001;34(5):386-389. DOI: 10.1046/j.1365-2591.2001.00259.x.

5. Martins JNR, DiBernardo J. Torsional failure characteristics of a NiTi file based on a case report. Rev Port Estomatol Med Dent Cir Maxilofac 2010;51(2):85-89. DOI: 10.1016/S1646-2890(10)70091-1.

6. Miyara K, Yahata Y, Hayashi Y, et al. The influence of heat treatment on the mechanical properties of $\mathrm{Ni}-\mathrm{Ti}$ file materials. Dent Mater J 2014;33(1):27-31. DOI: 10.4012/dmj.2013-196.

7. Mohammadi Z, Soltani MK, Shalavi S, et al. A review of the various surface treatments of NiTi instruments. Iran Endod J 2014;9(4): 235-240.

8. Ounsi HF, Nassif W, Grandini S, et al. Evolution of nickel-titanium alloys in endodontics. J Contemp Dent Pract 2017;18(11):1090-1096. DOI: 10.5005/jp-journals-10024-2181.

9. Ye J, Gao Y. Metallurgical characterization of M-Wire nickel-titanium shape memory alloy used for endodontic rotary instruments during low-cycle fatigue. J Endod 2012;38(1):105-107. DOI: 10.1016/ j.joen.2011.09.028.

10. Alapati SB, Brantley WA, lijima M, et al. Metallurgical characterization of a new nickel-titanium wire for rotary endodontic instruments. J Endod 2009;35(11):1589-1593. DOI: 10.1016/j.joen.2009.08. 004.

11. Pereira ES, Peixoto IF, Viana AC, et al. Physical and mechanical properties of a thermomechanically treated $\mathrm{NiTi}$ wire used in the manufacture of rotary endodontic instruments. Int Endod J 2012;45(5):469-474. DOI: 10.1111/j.1365-2591.2011.01998.x.
12. Duerig TW, Bhattacharya K. The influence of the R-phase on the superelastic behavior of NiTi. Shap Mem Superelasticity 2015;1: 153-161. DOI: 10.1007/s40830-015-0013-4.

13. Santos LD, Resende PD, Bahia MG, et al. Effects of R-phase on mechanical responses of a nickel-titanium endodontic instrument: structural characterization and finite element analysis. Sci World J 2016;2016:7617493. DOI: 10.1155/2016/7617493.

14. Bouska J, Justman B, Williamson A, et al. Resistance to cyclic fatigue failure of a new endodontic rotary file. J Endod 2012;38(5):667-669. DOI: 10.1016/j.joen.2012.01.016.

15. Singh $\mathrm{H}$, Kapoor P. Hyflex CM and EDM files: revolutionizing the art and science of endodontics. J Dent Health Oral Disord Ther 2016;5(7):385-387. DOI: 10.15406/jdhodt.2016.05.00182.

16. Shen Y, Zhou HM, Zheng YF, et al. Metallurgical characterization of controlled memory wire nickel-titanium rotary instruments. J Endod 2011;37(11):1566-1571. DOI: 10.1016/j.joen.2011.08.005.

17. Aoun $\mathrm{CM}$, Nehme WB, Naaman AS, et al. Review and classification of endodontic heat treatment procedures. Int J Curr Res 2017;9(5): 51300-51306.

18. Shen $Y, T r a C$, Hieawy A, et al. Effect of torsional and fatigue preloading on HyFlex EDM files. J Endod 2018;44(4):643-647. DOI: 10.1016/ j.joen.2017.12.002.

19. Pirani C, lacono F, Generali L, et al. HyFlex EDM: superficial features, metallurgical analysis and fatigue resistance of innovative electro discharge machined NiTi rotary instruments. Int Endod J 2016;49(5):483-493. DOI: 10.1111/iej.12470.

20. Gao Y, Gutmann JL, Wilkinson K, et al. Evaluation of the impact of raw materials on the fatigue and mechanical properties of ProFile Vortex rotary instruments. J Endod 2012;38(3):398-401. DOI: 10.1016/ j.joen.2011.11.004.

21. Silva EJ, Vieira VT, Belladonna FG, et al. Cyclic and torsional fatigue resistance of XP-endo Shaper and TRUShape instruments. J Endod 2018;44(1):168-172. DOI: 10.1016/j.joen.2017.08.033.

22. Silva EJ, Belladonna FG, Zuolo AS, et al. Effectiveness of XP-endo Finisher and XP-endo Finisher $\mathrm{R}$ in removing root filling remnants: a micro-CT study. Int Endod J 2018;51(1):86-91. DOI: 10.1111/iej.12788.

23. Pérez-Higueras JJ, Arias A, José C. Cyclic fatigue resistance of K3, K3XF, and twisted file nickel-titanium files under continuous rotation or reciprocating motion. J Endod 2013;39(12):1585-1588. DOI: 10.1016/ j.joen.2013.07.020

24. Uzunoglu E, Turker SA. Impact of different file systems on the amount of apically extruded debris during endodontic retreatment. Eur J Dent 2016;10(2):210-214. DOI: 10.4103/1305-7456.178306.

25. Hansen C, Torsion and Bending Properties of EdgeEndo Files. (2016). Master's Theses (2009-). Paper 353. http://epublications.marquette. edu/theses_open/353. 